\title{
Radiographic Evidence of an Epidural and Paraspinal Abscess after Lumbar Epidural Steroid Injection
}

\author{
Jamal Hasoon ${ }^{1 *}$, Amnon A Berger ${ }^{1}$, Ivan Urits ${ }^{1}$, Vwaire Orhurhu², Omar Viswanath ${ }^{3,4,5}$ \\ 1Department of Anesthesia, Beth Israel Deaconess Medical Center, Harvard Medical School, Boston, USA \\ 2Department of Anesthesia, Massachusetts General Hospital, Harvard Medical School, Boston, USA \\ 3Valley Anesthesiology and Pain Consultants - Envision Physician Services, Phoenix, USA \\ 4 Department of Anesthesiology, University of Arizona College of Medicine-Phoenix, USA \\ 5Department of Anesthesiology, Creighton University School of Medicine, USA \\ *Corresponding author: Jamal Hasoon MD, Beth Israel Deaconess Medical Center Department of Anesthesiology, Critical Care, and \\ Pain Medicine 330 Brookline Avenue, Boston, MA, USA
}

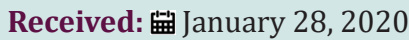

Published: 海 February 04, 2020

\section{Keywords: Epidural abscess; Lumbar spine; Complications; Chronic pain}

\section{Case Discussion}

Spinal epidural abscess (SEA) is a rare suppurative infection of the spinal cord. SEAs are formed in the true spinal epidural space between the dura and vertebral wall. They are the result of direct spread from adjacent structures, hematogenous spread or iatrogenic inoculation of bacteria into the spinal canal. SEA can lead to compressive symptoms causing pain, neurological deficits and even death. The pathogenesis of spinal damage is not only by direct compression but also through mechanisms such as thrombosis and ischemia resulting from interruption of arterial blood supply, and the effects of bacterial toxins and inflammatory mediators. SEAs are a rare disease process, making the actual incidence hard to assess. A single institution retrospective trial assessed the incidence at 5.1 out of 10,000 admissions; $52 \%$ of these had a source identified. Moreover, $26 \%$ of all admissions followed bacteremia, suggesting a hematogenous source in $25-50 \%$ of these patients [1]. While the disease incidence seems to be higher than expected, it is hard to determine if this is due to the increased numbers of neuraxial interventional procedures or increased diagnosis owing to increased availability and technical improvement of MRI scanners [2].

Diagnosis ultimately relies on identification of an abscess with MRI and is commonly delayed due to variation in presentation as very few patients present with the classic triad of fever, back pain and neurological deficits. A high level of suspicion must be maintained when a diagnosis is suspected. Risk factors for primary spinal infections include age $(<20$ and $>50)$, chronic disease (diabetes, cirrhosis, cancer, rheumatic disease), medical procedures, immunosuppression and intravenous drug use (IVDU) [3]. Staphylococcal species represent the majority of identified infectious causes for SEAs. While treatment has been historically surgicalin nature, advances in imaging has driven increasingly earlier diagnoses, allowing medical management of a select population of patients. A metanalysis compared medical and surgical treatment in a historic cohort of patients. This analysis identified a significant increase in medical management reaching $40 \%$ in this series. While there was no identified difference in prognosis between medical and surgical management, patients presenting with neurological deficits were more likely to be managed surgically. The analysis identified diabetes, elevated CRP, marked leukocytosis, positive blood cultures, age $>65$ years, methicillin-resistant Staphylococcus aureus (MRSA) infection, and advanced neurological deficits as risk factors for failed medical management [4]. Similar results were obtained by another systematic review and it also demonstrated that delayed surgical management, when indicated, results in worse prognosis [5]. 
Here we provide radiographic evidence of a 66-year-old patient with diabetes and hepatocellular carcinoma who suffered from a spinal epidural abscess believed to be caused by a lumbar epidural steroid injection from an outside pain provider one year prior. The patient initially presented with severe debilitating back pain and fevers one week after the intervention. He was diagnosed at an outside hospital and his treatment included medical management with IV antibiotics as well as serial drainage procedures by interventional radiology. He failed to respond and ultimately underwent multiple neurosurgical procedures for decompression and drainage which ultimately treated the infection. The condition left him nearly paraplegic with minimal strength in his lower extremities and debilitating back pain. The images demonstrate chronic inflammatory changes and fluid collections from the infection as well as endplate destruction around the L1-L2 levels (Figure 1).

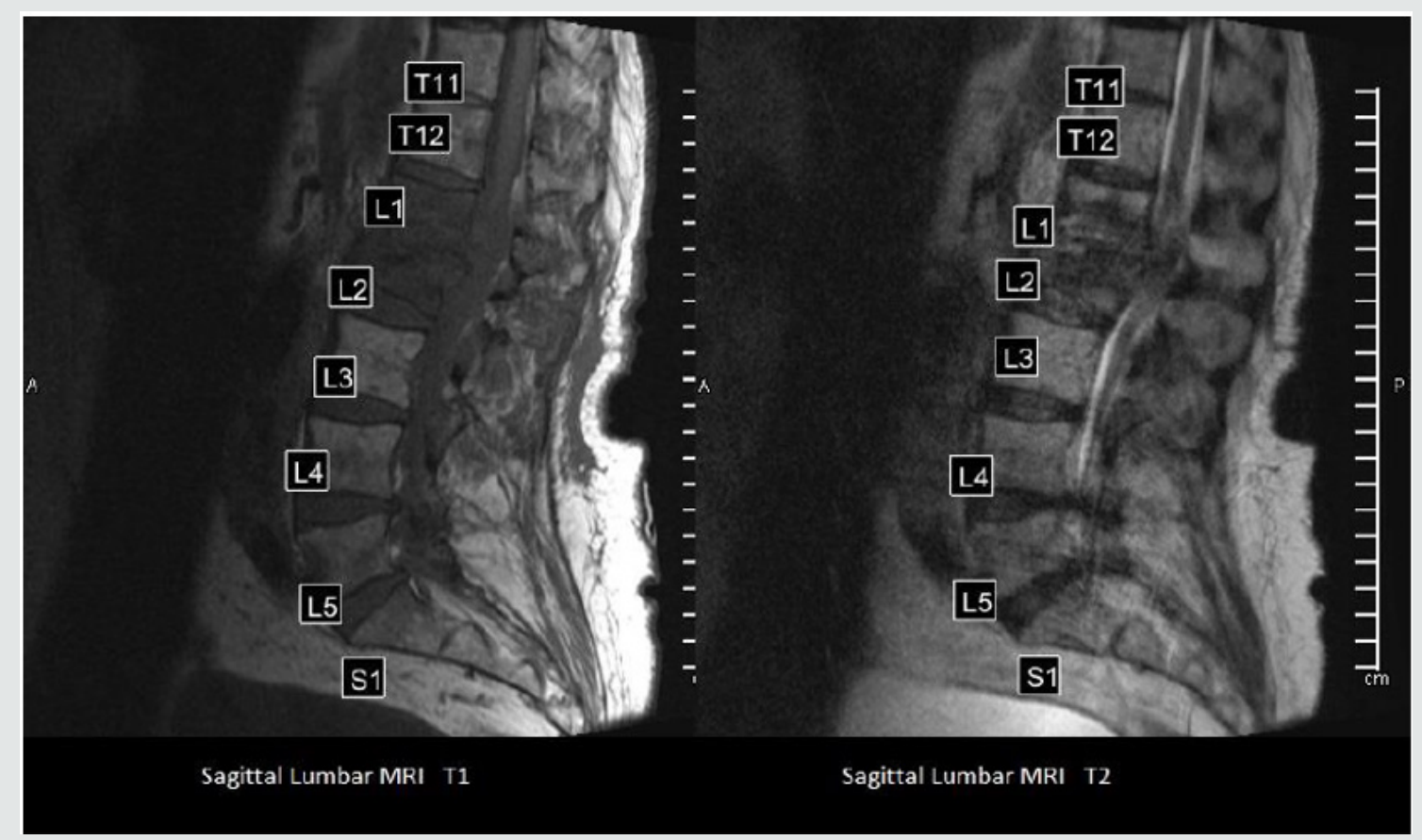

Figure 1: Sagittal Lumbar MRIs that demonstrate chronic inflammatory changes and fluid collections from the infection as well as endplate destruction around the L1-L2 levels.

- $\quad$ Conflicts of Interest: The authors declare that there are no conflicts of interest.

- $\quad$ Sources of financial support that require acknowledgment (if any): None

- Type of contribution of the authors: Concept and design: $\mathrm{JH}, \mathrm{AB}$; Drafting the manuscript: JH, AB; Literature Review: JH, AB, IU, VO, OV; Critical Revision: JH, IU, VO, OV

- Approval code issued by the institutional review board (IRB): Approval code: unnecessary Acknowledgements: None

- Informed consent: Obtained from the patient

\section{References}

1. Vakili M, Crum Cianflone NF (2017) Spinal epidural abscess: a series of 101 cases. The American journal of medicine 130(12): 1458-1463.
2. Sørensen P (2003) Spinal epidural abscesses: conservative treatment for selected subgroups of patients. British journal of neurosurgery 17(6): 513-518.

3. Gregori F, Grasso G, Iaiani G (2019) Treatment algorithm for spontaneous spinal infections: A review of the literature. Journal of craniovertebral junction \& spine 10(1): 3-9.

4. Arko L, Quach E, Nguyen V, Chang D, Sukul V, et al. (2014) Medical and surgical management of spinal epidural abscess: a systematic review. Neurosurgical focus 37(2): E4.

5. Wang TY, Harward SC, Tsvankin V, Bell H, Charalambous L, et al. (2019) Neurological Outcomes After Surgical or Conservative Management of Spontaneous Spinal Epidural Abscesses. Clinical spine surgery 32(1): $18-29$. 


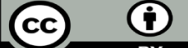

This work is licensed under Creative Commons Attribution 4.0 License

To Submit Your Article Click Here:

Submit Article

DOI: 10.32474 /GJAPM.2020.02.000146

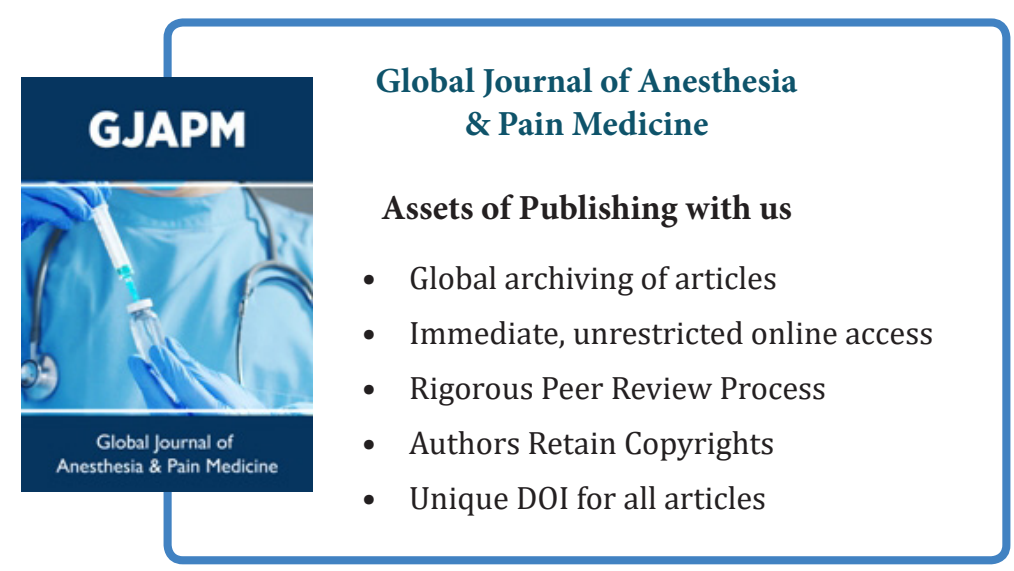

